\title{
Nonlinear Cascaded Femtosecond Third Harmonic Generation by Multi-grating Periodically Poled MgO-doped Lithium Niobate
}

\author{
Shuanggen Zhang ${ }^{1 *}$, Wenchao You ${ }^{1}$, Zhangchao Huang ${ }^{2}$ \\ ${ }^{1}$ School of Electronic Information Engineering, Tianjin University of Technology, Tianjin Key Laboratory of Film \\ Electronic and Communication Device, Engineering Research Center of Communication Devices Ministry of Education, \\ Tianjin University of Technology, Tianjin, China \\ ${ }^{2}$ School of Physics, Nankai University, Tianjin, China \\ Email: *shgzhang@tjut.edu.cn
}

Received 2013

\begin{abstract}
Nonlinear cascaded femtosecond third harmonic generation was experimentally investigated pumped by 100 fs pulses at optical communication band $1550 \mathrm{~nm}$ using a multi-grating $5 \mathrm{~mol}$ \% MgO-doped periodically poled lithium niobate crystal. The optimized efficiency of $10.8 \%$ was achieved with the simultaneous phase-matching of the second harmonic and sum frequency process. And the third harmonic spectrum reached as broad as $8.7 \mathrm{~nm}$ because of the choosing of a small group velocity mismatching between the fundamental and second harmonic pulses. Nonlinear cascaded method will provide a reference for the efficient frequency conversion in the high intensity range.
\end{abstract}

Keywords: Femtosecond; Third Harmonic Generation; Second Harmonic Generation; Cascaded Nonlinear Process

\section{Introduction}

Frequency upconversion is a useful nonlinear process to achieve short wavelength, and third harmonic generation (THG) is one of the most effective upconversion process, which can be used to generate optical emission with shorter wavelength. Generally, third-order susceptibility of material contributes to THG process, i. e., direct THG. Besides direct THG method, cascaded THG by second harmonic and sum frequency process is a more efficient method. Higher conversion efficiency can be achieved with quadratic nonlinearity using two cascaded nonlinear processes.

However, the most efficient conversion of cascaded THG is achieved when the phase-matching conditions for both second harmonic and sum frequency processes are satisfied. In case of $\mathrm{cw}$ or quasi-cw regimes, the simultaneous phase-matching of the two parametric processes has been realized in several different structures in the past two decades [1-3]. For two-dimension nonlinear photonic crystals, they are often utilized to realize the noncollinear THG [4-6]. Recently, collinear THG was also achieved by a short-range-ordered two-dimension nonlinear photonic crystal [7]. However, there are few reports on cascaded THG of ultrashort pulses. N. Fujioka realized noncollinear cascaded THG of femtosecond

\footnotetext{
${ }^{*}$ Corresponding author.
}

pulses using two-dimension periodically poled lithium niobate and THG efficiency of $8 \%$ with spectral width of $4 \mathrm{~nm}$ was obtained [8].

In this paper, we demonstrated a collinear cascaded THG of femtosecond pulses with high intensity in a 5 mol. \% MgO-doped periodically poled lithium niobate crystal. In the single pass scheme, the optimal THG efficiency of $10.8 \%$ was obtained at the input intensity of 74 $\mathrm{GW} / \mathrm{cm}^{2}$. The TH spectral width reached as broad as 8.7 $\mathrm{nm}$ with a small group velocity mismatch (GVM) between fundamental pulses and SH pulses.

\section{Experimental Configuration}

In general, a periodic QPM material can provide only one effective wave vector for a parametric process. However, a QPM material with a period of $\Lambda$ is also possible to provide two effective wave vectors contributing to the two QPM processes, respectively. In such a periodic QPM marital, the cascaded THG, which involves the simultaneous SHG and SFG processes, with the respective $\Delta k_{I}$ and $\Delta k_{I I}$ as follows:

$$
\begin{aligned}
\Delta k_{I} & =k_{2}-2 k_{1}-k_{Q P M 1} \\
& =4 \pi\left(n_{2}-n_{1}\right) / \lambda_{1}-m_{1} 2 \pi / \Lambda \\
\Delta k_{I I} & =k_{3}-k_{2}-k_{1}-k_{Q P M 2} \\
& =2 \pi\left(3 n_{3}-2 n_{2}-n_{1}\right) / \lambda_{1}-m_{2} 2 \pi / \Lambda
\end{aligned}
$$


where the subscripts 1,2 , and 3 represent the fundamental, $\mathrm{SH}$ and TH waves, respectively. $k_{i}$ and $n_{i}$ are the wave vector and the refractive index for the $i$-th wave ( $I$ $=1,2,3), k_{\mathrm{QPM} 1}$ and $k_{\mathrm{QPM} 2}$ are the QPM wave vectors of $m_{1}$ and $m_{2}$ orders for SHG and SFG, respectively.

From the Sellmeier equations of $5 \mathrm{~mol}$ \% $\mathrm{MgO}$-doped congruent $\mathrm{LiNbO}_{3}$ [9], it is found that optimal grating period of $20.37 \mu \mathrm{m}$ can be used to achieve an efficient cascaded THG under $35^{\circ} \mathrm{C}$, provided that the polarization of the fundamental wave is chosen to be ordinary, and the polarizations of the $\mathrm{SH}$ and $\mathrm{TH}$ waves are chosen to be extraordinary. For the femtosecond pulses with a large spectral width, it has a large phase-matching bandwidth in the frequency conversion [10]. Therefore, the efficient SFG can still be achieved with the existence of a small wave-vector mismatch.

Considering the refractive index changes induced by the nonlinearity caused by the high intensity [11], the QPM conditions may be slightly changed with respect to the above estimations. To overcome this problem, the MgO: PPLN sample in our experiment (made by $\mathrm{HC}$ Photonics Corporation) was composed of ten parallel periodically-poled structures with different periods around $20.37 \mu \mathrm{m}$ (from $19.5 \mu \mathrm{m}$ to $21.3 \mu \mathrm{m}$ with the interval of $0.2 \mu \mathrm{m}$ ). The length and the thickness of the sample were $5 \mathrm{~mm}$ and $0.5 \mathrm{~mm}$, respectively. Its two end surfaces were optically flat polished but uncoated. The polarizations of ordinary beam and extraordinary beam are chosen to be parallel to $\mathrm{y}$-axis and parallel to $\mathrm{z}$-axis, respectively. The $\mathrm{x}$-axis was chosen as the propagation direction. The polarization of the ordinary fundamental wave was set to be parallel to y-axis.

The experimental schematic is shown in Figure 1. The fundamental light source was an optical parametric amplifier, pumped by regeneratively amplified Ti: sapphire laser, operated at a repetition rate of $1 \mathrm{kHz}$. The fundamental pulses at a central wavelength of $1550 \mathrm{~nm}$ had a pulse duration about 100 fs and a spectral width (FWHM) about $60 \mathrm{~nm}$. A high-transmission mirror at $1550 \mathrm{~nm}$ behind the laser source was used to inhibit other wavelengths. A combination of a half-wave plate at $1550 \mathrm{~nm}$ and a Glen-Taylor prism was used to adjust the power of fundamental wave. When the Glen-Taylor prism is rotated to the right angle, the ordinary beam is allowed to pass only. A lens with a focal length of $200 \mathrm{~mm}$ is used to couple the fundamental beam into the sample. To avoid the crystal damage caused by the high intensity, we set the focus at a distance of $15 \mathrm{~mm}$ behind the output face of the crystal. The beam waist at the focus is about $50 \mu \mathrm{m}$. The radii of the beam in the input and output faces of the sample are 203.6 and $156.2 \mu \mathrm{m}$, respectively. The crystal was placed inside a temperature-controlled oven, in which the operation temperature can be controlled up to $200^{\circ} \mathrm{C}$ with an accuracy of $0.1^{\circ} \mathrm{C}$. Behind the oven, a focus lens, a high-reflecting mirror for the residual fundamental wave, and a band-pass filter for the $\mathrm{SH}$ or $\mathrm{TH}$ wave were used.

\section{Results and Discussions}

The dependences of the directly measured $\mathrm{SH}$ and $\mathrm{TH}$ power on the input power are shown in Figure 2(a). Under our experimental condition, the input power of 10 $\mathrm{mW}$ corresponds to an input peak intensity of 100 $\mathrm{GW} / \mathrm{cm}^{2}$. The maximum input power is $54 \mathrm{~mW}$, corresponding to an input peak intensity of $540 \mathrm{GW} / \mathrm{cm}^{2}$. The highest $\mathrm{SH}$ efficiency of $4.5 \%$ is obtained at the input power of $13.3 \mathrm{~mW}$ (an input peak intensity of 133 $\mathrm{GW} / \mathrm{cm}^{2}$ ), while the highest TH efficiency of $10.8 \%$ is obtained at the input power of $7.4 \mathrm{~mW}$ (an input peak intensity of $74 \mathrm{GW} / \mathrm{cm}^{2}$ ). When the losses are taken into account, composed of the coupling loss of $5 \%$ and the Fresnel losses $(14.2 \%$ for the fundamental wave, $13.6 \%$ for the $\mathrm{SH}$ wave, $14.5 \%$ for the $\mathrm{TH}$ wave), the highest efficiencies of the generated $\mathrm{SH}$ and $\mathrm{TH}$ waves are $6.5 \%$ and $15.7 \%$, respectively, as shown in Figure 2(b). As the input power increases, the $\mathrm{SH}$ power increases linearly and the TH power increases quadratically. The THG efficiency saturation has been observed at the intensity level of $20 \mathrm{GW} / \mathrm{cm}^{2}$ in the femtosecond cascaded THG [8]. When the intensity is raised to the order of magnitude of $100 \mathrm{GW} / \mathrm{cm}^{2}$, the THG efficiency can't keep constant. It decreases with the increasing input intensity.

The spectra were measured by a high-resolution spectrometer (Ocean Optics), as shown in Figure 3(a). The $\mathrm{SH}$ spectrum has a smooth profile with a FWHM of $4.7 \mathrm{~nm}$. The main peak of the TH spectrum appears at $508.9 \mathrm{~nm}$

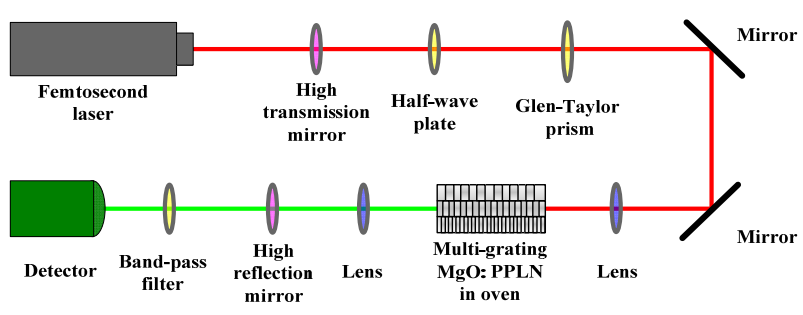

Figure 1. Experimental setup schematic.

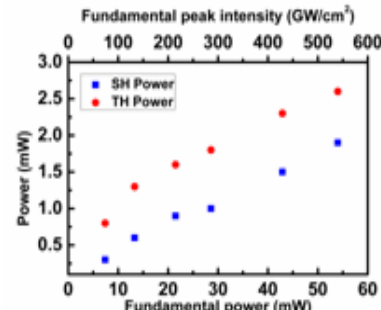

(a)

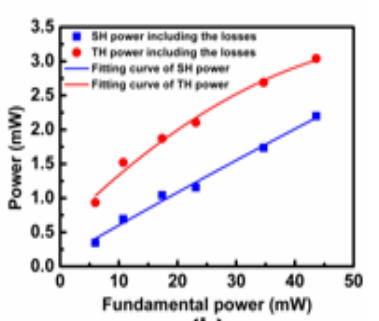

(b)
Figure 2. (a) Measured SH and TH power versus fundamental power; (b) SH and TH power including losses and fitting curves. 

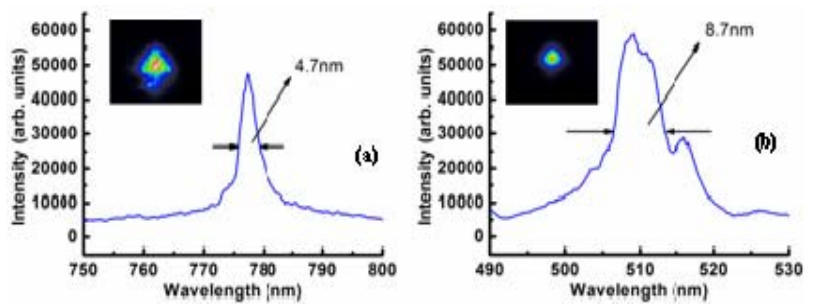

Figure 3. (a) SH spectra and mode; (b) TH spectra and mode.

with a FWHM of $8.7 \mathrm{~nm}$ and another peak locates at $516.1 \mathrm{~nm}$. The TH spectral width is more than two times as the reported value of $4 \mathrm{~nm}$ by the cascaded THG of 118 fs pump pulses with a FWHM of $51 \mathrm{~nm}$ in the two-dimension PPLN [8]. The measured TH mode in the output face of the sample is shown in inset of Figure 3(b). A nearly circular profile indicates a good spatial intensity distribution of THG.

During the cascaded processes, the TH bandwidth is limited by GVM among fundamental, $\mathrm{SH}$ and $\mathrm{TH}$ pulses. However, the GVM between the fundamental and SH pulses is the most important because it directly determines the effective interaction length of the SFG process [8]. In our experiment, the small GVM between the fundamental and SH pulses mainly results in the generation of the broadband TH wave. We choose the SHG type $(\mathrm{o}+\mathrm{o}-\mathrm{e})$ that the fundamental and $\mathrm{SH}$ pulses have different polarizations. In the $5 \mathrm{~mol}$. \% MgO-doped congruent lithium niobate crystal, the GVM between ordinary fundamental wave $(1550 \mathrm{~nm})$ and extraordinary $\mathrm{SH}$ wave $(775 \mathrm{~nm})$ is only $155.6 \mathrm{fs} / \mathrm{cm}$, while that is 3034 $\mathrm{fs} / \mathrm{cm}$ for the SHG type (e+e--e) that the polarizations of the fundamental and SH pulses are both extraordinary [12].

\section{Conclusions}

Efficient cascaded femtosecond THG using a 5 mol. \% $\mathrm{MgO}$-doped multi-grating periodically poled crystal was experimentally demonstrated. The optimal TH efficiency of $10.8 \%$ was obtained in case of simultaneous phase matching of SHG and the SFG. The TH spectral width reached as abroad as $8.7 \mathrm{~nm}$ with a small GVM between the fundamental and SH pulses. Such nonlinear cascaded process will provide a reference for the efficient frequency conversion in the high intensity range.

\section{Acknowledgements}

This work was supported by the National Natural Science Foundation of China (11004152), Program of Tianjin Municipal Education Commission (20090715).

\section{REFERENCES}

[1] K. Kintaka, M. Fujimura, T. Suhara and H. Nishihara, "Third Harmonic Generation of Nd: YAG Laser Light in
Periodically Poled $\mathrm{LiNbO}_{3}$ ", Electronic Letters, Vol. 33, No. 17, 1997, pp. 1459-1461. doi:10.1049/el:19970990

[2] S. N. Zhu, Y. Y. Zhu and N. B. Ming, "Quasi-PhaseMatched Third Harmonic Generation in a Quasi- Periodic Optical Superlattice," Science, Vol. 278, No. 5339, 1997, pp. 843-846. doi:10.1126/science.278.5339.843

[3] C. Zhang, H. Wei, Y. Y. Zhu, H. T. Wang, S. N. Zhu and N. B. Ming, "Third-Harmonic Generation in A General Two-Component Quasi-Periodic Optical Superlattice," Optics Letters, Vol. 26, No. 12, 2001, pp. 899-901. doi:10.1364/OL.26.000899

[4] N. G. R. Broderick, R. T. Bratfalean, T. M. Monro, D. J. Richardson and C. M. de Sterke, "Temperature and Wavelength Tuning of Second-, Third-, and Fourth-Harmonic Generation in A Two-Dimensional Hexagonally Poled Nonlinear Crystal," Journal of the Optical Society of America B, Vol. 19, No. 9, 2002, pp. 2263-2272. doi:10.1364/JOSAB.19.002263

[5] A. H. Norton and C. M. de Sterke, "Optimal Poling of Nonlinear Photonic Crystals for Frequency Conversion," Optics Letters, Vol. 28, No. 3, 2003, pp. 188-190.

[6] A. H. Norton and C. M. de Sterke, "Two-Dimensional Poling Patterns for 3rd and 4th Harmonic Generation," Optics Express, Vol. 11, No. 9, 2003, pp. 1008-1014. doi:10.1364/OE.11.001008

[7] Y. Sheng, S. M. Saltiel and K. Koynov, "Cascaded Third-Harmonic Generation in A Single Short-Range -Ordered Nonlinear Photonic Crystal," Optics Letters, Vol. 34, No. 5, 2009, pp. 656-658. doi:10.1364/OL.34.000656

[8] N. Fujioka, S. Ashihara, H. Ono, T. Shimura and K. Kuroda, "Cascaded Third-Harmonic Generation of Ultrashort Optical Pulses in Two-Dimensional Quasi-PhaseMatching Gratings," Journal of the Optical Society of America B, Vol. 24, No.9, 2007, pp. 2394-2405. doi:10.1364/JOSAB.24.002394

[9] D. E. Zelmon, D. L. Small and D. Jundt, "Infrared Corrected Sellmeier Coefficients for Congruently Grown Lithium Niobate and 5 mol.\% Magnesium Oxide -Doped Lithium Niobate," Journal of the Optical Society of America B, Vol. 14, No. 12, 1997, pp. 3319-3322. doi:10.1364/JOSAB.14.003319

[10] Z. C. Huang, C. H. Tu, S. G. Zhang, Y. N. Li, F. Y. Lu, Y. $\mathrm{X}$. Fan and E. B. Li, "Femtosecond Second-Harmonic Generation in Periodically Poled Lithium Niobate Waveguides Written by Femtosecond Laser Pulses," Optics Letters, Vol. 35, No. 6, 2010, pp. 877-879. doi:10.1364/OL.35.000877

[11] DeSalvo, A. A. Said, D. J. Hagan, E. W. Van Stryland, and M. Sheik-Bahae, "Infrared to Ultraviolet Measurements of Two-Photon Absorption and $\mathrm{N}_{2}$ in Wide Bandgap Solids," IEEE Journal of Quantum Electronics, Vol.32, No. 8, 1996, pp. 1324-1333. doi: $10.1109 / 3.511545$

[12] N. E. Yu, J. H. Ro, M. Cha, S. Kurimura and T. Taira, "Broadband Quasi-Phase-Matched Second-Harmonic Generation in $\mathrm{MgO}$-Doped Periodically Poled $\mathrm{LiNbO}_{3}$ at the Communications Band," Optics Letters, Vol. 27, No. 12, 2002, pp. 1046-1048. doi:10.1364/OL.27.001046 